\title{
miR-17-3P regulates the proliferation and survival of colon cancer cells by targeting Par4
}

\author{
DEBAO LU ${ }^{1}$, LIANG TANG $^{2}$, YAN ZHUANG $^{2}$ and PENG ZHAO ${ }^{2}$ \\ ${ }^{1}$ Department of General Surgery, Tianjin TEDA Hospital, Tianjin 300457; ${ }^{2}$ Department of Colorectal Tumor, \\ Tianjin Medical University Cancer Institute and Hospital, Tianjin 300202, P.R. China
}

Received December 19, 2016; Accepted September 1, 2017

DOI: $10.3892 / \mathrm{mmr} .2017 .7863$

\begin{abstract}
Colorectal cancer (CRC) is a common malignancy worldwide. However, the pathogenesis by which CRC progression occurs remains to be elucidated. The present study investigated the role of miRNA (miR)-17-3P in the regulation of CRC cell survival. Firstly, miR-17-3P expression was aberrantly upregulated in human CRC tumor tissues compared with controls. Further results demonstrated that the proliferation capacity of human CRC SW480 and LoVo cells was significantly increased by an miR-17-3P specific mimic, and was inhibited by miR-17-3P silencing. Conversely, the apoptosis of human CRC cells was remarkably decreased by miR-17-3P mimic, and enhanced by miR-17-3P suppression compared with control. Additionally, it was observed that there was a potential binding site of miR-17-3P on the 3'-untranslated region of Prostate apoptosis responde-4 (Par4) and miR-17-3P may directly target Par4 mRNA. In human CRC cells, an miR-17-3P inhibitor significantly upregulated Par 4 expression, however the miR-17-3P mimic reduced Par4expression. Furthermore, Par4 expression exhibited an inhibitory effect on the proliferation of CRC cells transfected with miR-17-3P mimic, and exhibited a promoting role in the repressed apoptosis by miR-17-3P mimic. Inconclusion, the results of the present study demonstrated that miR-17-3P is important in CRC cell survival by targeting Par4, indicating a novel finding regarding human CRC progression.
\end{abstract}

\section{Introduction}

Colorectal cancer (CRC) is a common malignancy around the world. CRC is the result of multistep processes in which the sequential mutations of oncogenes or tumor suppressor

Correspondence to: Dr Peng Zhao, Department of Colorectal Tumor, Tianjin Medical University Cancer Institute and Hospital, Huanhu Xi Road, Hexi, Tianjin 300202, P.R. China

E-mail: pz_1967223@163.com

Keywords: miR-17-3P, prostate apoptosis responde-4, colorectal cancer, proliferation, apoptosis genes and chromosomal instability are considered as the main oncogenic factors (1). According to the difference of clinic pathological entities, CRC can be classified as various histological subtypes, including sporadic, familial, and hereditary forms. In the CRC cases, different CRC form exhibits different proportion. In general, the sporadic form are most CRC form. 10-30\% of CRC cases correspond to the familial form, while hereditary is the least (2). In the treatment of CRC, early detection and surgical resection are the primary approach. Although there are significant improvements in the diagnostic method and surgical therapy, the cure rate of patients with CRC is very poor $(1,3)$. Therefore, furthermore knowledge about the pathology of CRC progression are urgently needed to improve the therapeutic strategies for human CRC.

As endogenous regulators, microRNAs (miRNAs) is a family of non-coding 19-22 nucleotides small single-stranded RNAs molecules, and exert key roles in the expression of genes (4). It has been recognized that dysregulation of miRNAs expression is found and proven to be implicated in various human diseases, including glioma, metastatic prostate carcinoma and other cancers $(5,6)$. In terms of CRC, aberrant regulation of miRNAs has been reported widely in patients and is associated with the pathogenesis of human CRC, miR-29a, miR-135b, miR-18b, miR-124, miR-222 and miR-31 (7-9). Members of miR-17-92 cluster has been proven to be commonly dysregulated in various cancers and plays role in the development and maintenance of tumor (10). In the present study, we determined that miR-17-3P expression is aberrantly regulated in human CRC cell model.

Prostate apoptosis responde-4 (Par4), also known as PAWR, is a WT-1-interacting protein in variety of tissues, and is required as a tumor suppressor (11). Par4 has a critical role in cell apoptosis. Accumulating evidences suggest that lowly expressed Par4 is associated with the tumorigenesis of human cancers (12-14). However, whether the dysfunction of PAR4 is involved in the progression of CRC is still unexplored. Herein, we propose that miR-17-3P contributes to the pathogenesis of human CRC by targeting Par4. miR-17-3P expression was found to be abnormally expressed in human CRC cells, and exerts regulatory roles in the survival of CRC cells by directly targeting Par4. These results may provide a novel finding about human CRC. 


\section{Materials and methods}

Ethics statement. All patients gave the informed consent. This study was approved by the Ethics Committee of Tianjin Medical University.

CRC tissues and cells. Primary CRC biopsies and adjacent tissues were collected from 40 patients undergoing surgery resection at Tianjin Medical University. CRC cell lines SW480, LoVo and HCT-116 were obtained from ATCC (Manassas, VA, USA). NCM460 normal colon epithelial cells were obtained from Rongbai Biotechnology, Co., Ltd., (Shanghai, China). HCT-116 cells were maintained in McCoy's 5a medium, LoVo cells were maintained in F12-K medium and SW480 cells were cultured in DMEM medium containing $10 \% \mathrm{FBS}$ at $37^{\circ} \mathrm{C}$. NCM460 cells were maintained in DMEM-H medium as the control.

Cell transfection. Using Lipofectamine 2000 (Invitrogen, Carlsbad, CA, USA), 100 nM miR-17-3P mimic, inhibitor or negative control miRNA (RiboBio Co., Ltd., Guangzhou, China) were transfected into cultured cells in accord with manufacturer's instructions. The plasmid of Par4 (kindly provided by Dr Sun) was also transfected into cultured cells in absence or presence of miR-17-3P mimic. After $48 \mathrm{~h}$, the transfection efficacy was evaluated by RT-PCR and western blotting.

Western blot analysis. All proteins were collected from cell lysates and counted by bicinchoninic acid (BCA) assay. Then equal amount of proteins were separated by SDS-PAGE, and transfered to PVDF membrane. Primary antibodies were incubated with the blots (mouse anti-Par4 1:2,000 and mouse anti- $\beta$-actin 1:2,000 dilution; Abcam, Cambridge, MA, USA) overnight at $4^{\circ} \mathrm{C}$ temperature. The blots were probed by specific secondary antibodies for $1 \mathrm{~h}$ at RT, then performed by visualization with ECL.

Quantitative RT-PCR. Total RNA in tissues or cells were collected by Trizol reagent (Sigma-Aldrich, St. Louis, MO, USA). Expression of miR-17-3P and Par4 were detected using microRNA First-Strand Synthesis and miRNA Quantitation kits and CellAmp Direct RNA Prep kit (Takara, Dalian, China), respectively. The reaction: $95^{\circ} \mathrm{C}$ for $10 \mathrm{~min} ; 40$ cycles of $95^{\circ} \mathrm{C}$ for $1 \mathrm{~min}, 63^{\circ} \mathrm{C}$ for $2 \mathrm{~min}, 72^{\circ} \mathrm{C}$ for $1 \mathrm{~min}$; final $72^{\circ} \mathrm{C}$ for $10 \mathrm{~min}$. $\mathrm{Cq}$ values of $\mathrm{U} 6$ and GAPDH expression in tissues or cells were used as the internal control, respectively.

Cell apoptosis. Cell apoptosis assay was evaluated by annexin V-fluorescein isothiocyanate (FITC) assay. After $48 \mathrm{~h}$ of transfection, transfected cells $\left(5 \times 10^{6}\right)$ were seeded in the DMEM medium (serum-free) for another $12 \mathrm{~h}$, followed by harvesting using ice-cold PBS and re-suspending by binding buffer. Cells were then treated with $0.6 \mu \mathrm{g} / \mathrm{ml}$ annexin V-FITC and $0.5 \mu \mathrm{g} / \mathrm{ml}$ PI for $15 \mathrm{~min}$ in dark, and the apoptosis rate of cells were analyzed by the FACS Calibur ${ }^{\mathrm{TM}}$ system (BectonDickinson, San Jose, CA, USA).

Cell proliferation assay. After $48 \mathrm{~h}$, transfected cell proliferation was tested by CCK-8/WST-8 (Bioroot, Shanghai, China) in accordance with manufacturer's instructions. Briefly,
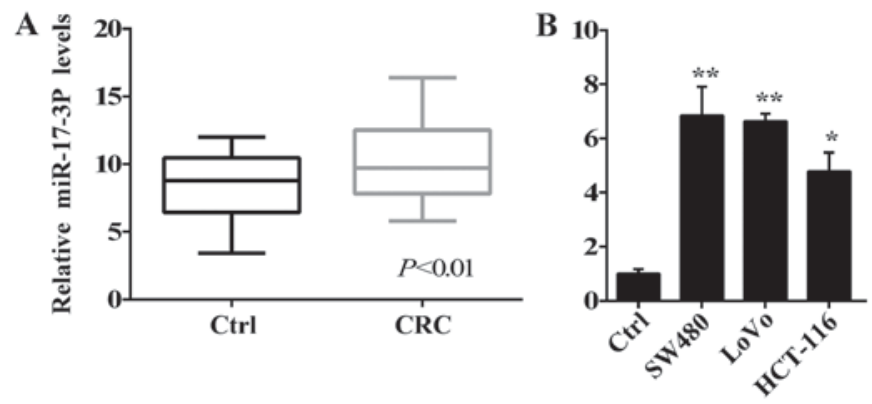

Figure 1. miR-17-3P expression in CRC tumor tissues and cells. (A) The miR-17-3P expression was measured in CRC tumor tissues as well as adjacent colonic tissues ( $\mathrm{Ctrl})\left({ }^{*} \mathrm{P}<0.05\right.$ vs. $\left.\mathrm{Ctrl}\right)$. (B) The miR-17-3P expression were measured in human CRC SW480, HCT-116 and LoVo cells as well as the normal colon epithelial cells NCM460 (Ctrl) $\left({ }^{*} \mathrm{P}<0.05,{ }^{* *} \mathrm{P}<0.01\right.$ vs. $\left.\mathrm{Ctrl}\right)$.

after $48 \mathrm{~h}$, transfected cells were planted in the 96-well plates (6x10 4 cells $/ \mathrm{ml}$ ). $10 \mu \mathrm{l}$ CCK- 8 solution was then added into the cells at 12, 24, 48 and $72 \mathrm{~h}$. Cells were maintained for $4 \mathrm{~h}$ and measured at $450 \mathrm{~nm}$.

Luciferase reporter assay. The assay was performed as described previously (15). In brief, 3'UTR of Par4 mRNA was cloned into pGL3 vector (Promega, Madison, WI, USA). QuikChange Lightning Site-Directed Mutagenesis kit (Stratagene) was applied to introduce the site-directed mutagenesis into predicted miR-17-3P binding site on mRNA of Par4. The recombinant vectors were then transfected into cultured CRC cells in the absence or presence of miR-17-3P mimic for $36 \mathrm{~h}$. And the luciferase activity was then tested by Dual Luciferase Assay (Promega).

Statistical analysis. Data were expressed as mean \pm SEM. SPSS15.0 was used to analyze the differences between groups by one-way ANOVA. $\mathrm{P}<0.05$ was considered to indicate a statistically significant difference.

\section{Results}

miR-17-3P is up-regulated in human CRC tissues and cells. We firstly identified miR-17-3P expression in tumor tissues of patients with CRC to determine the potential role of miR-17-3P in human CRC. As seen in Fig. 1A, miR-17-3P was significantly increased in tumor tissues of patients with CRC, while it was maintained at relatively low expression levels in adjacent colonic tissues. Further, we investigated the levels of miR-17-3P expression in human CRC SW480, HCT-116 and LoVo cells. As seen in Fig. 1B, miR-17-3P expression were aberrantly up-regulated in the SW480, HCT-116 and LoVo cells compared with the colon epithelial cells NCM460. Taken together, miR-17-3P expression may have a positive effect on the tumorigenesis of human CRC.

miR-17-3P promotes the survival of human CRC cells. We then employed the specific mimic and inhibitor of miR-17-3P to further identify the possible effect of high miR-17-3P levels on human CRC cells. According to the difference in expression of miR-17-3P, SW480 and LoVo cells were used as cell models in the subsequent experiments. Efficiency 
A

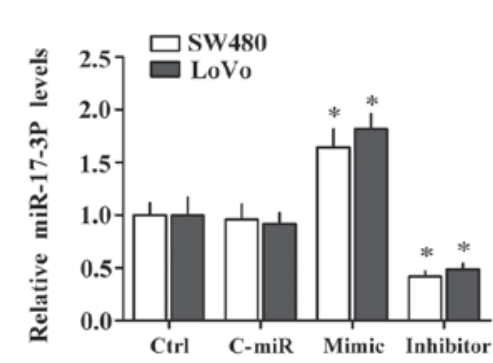

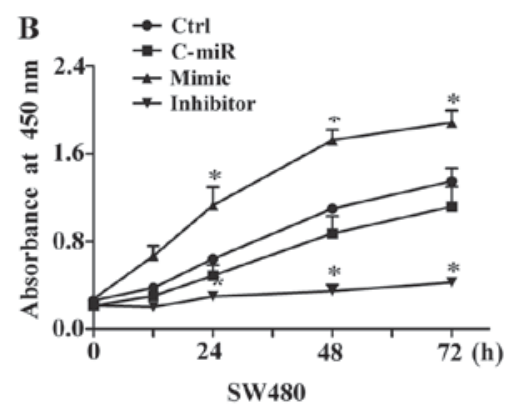
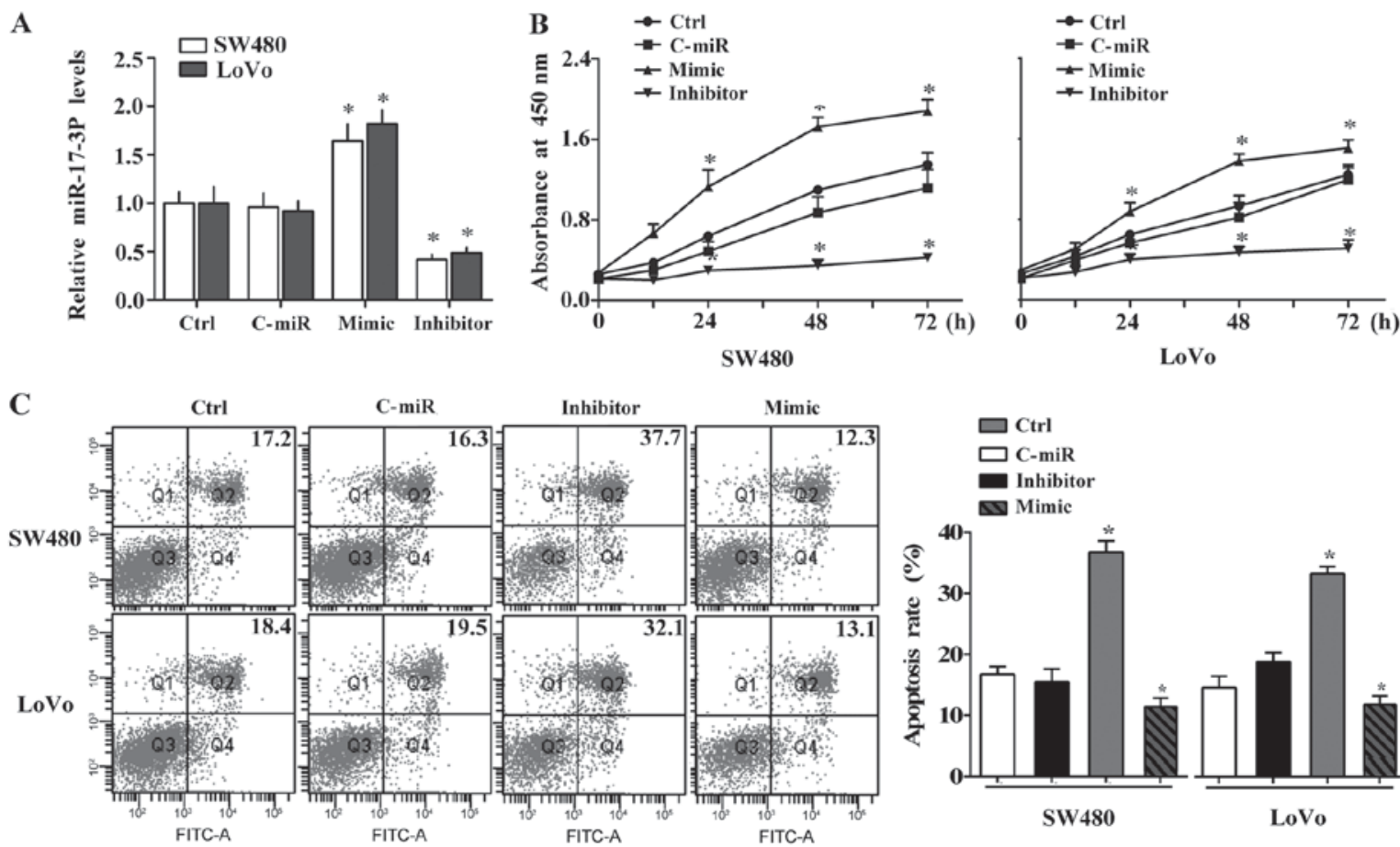

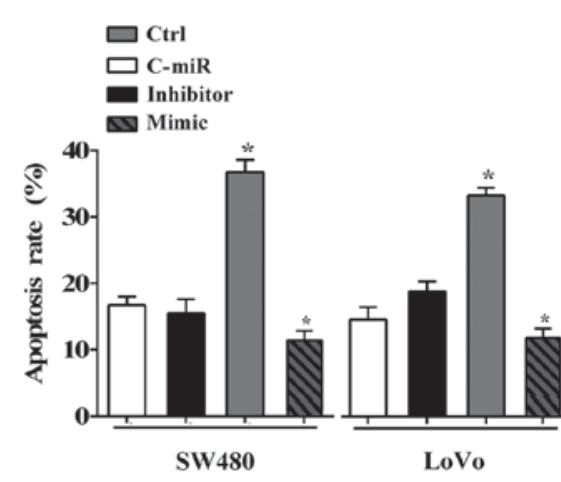

Figure 2. miR-17-3P promotes the survival of human colorectal cancer cells. SW480 and LoVo cells were transfected by miR-17-3P inhibitor, mimic or negative miRNA (C-MiR). (A) After $48 \mathrm{~h}$ of transfection, miR-17-3P expression was tested by RT-PCR assay in cells. (B) CCK-8 assays were performed to detect the proliferation of cells at 12, 24, 48 and $72 \mathrm{~h}$, respectively. (C) Flow cytometric analysis was performed to evaluate the apoptosis rate of transfected cells $($ ( $\mathrm{P}<0.05$ vs. Ctrl).

of the mimic and inhibitor were confirmed using RT-PCR assay. The specific mimic significantly increased, while the inhibitor inhibited, miR-17-3P expression in both SW480 and LoVo cells compared to the negative control miRNA (Fig. 2A).

In the CCK- 8 assay, the proliferation of CRC cells were examined. As shown in Fig. 2B, the proliferation were remarkably increased by miR-17-3P mimic in SW480 and LoVo cells compared to the negative control, while miR-17-3P silencing significantly suppressed the proliferation capacity of these two CRC cells. Furthermore, we investigated the apoptosis levels of CRC cells. miR-17-3P inhibition increased but miR-17-3P overexpression inhibited the apoptosis of SW480 and LoVo cells compared to control (Fig. 2C). These data imply that miR-17-3P may contribute to the human $\mathrm{CRC}$ progression by regulating the survival of CRC cells.

Par4 is the target of $m i R-17-3 P$. Par4 is a tumor suppressor in various cancers, including human CRC (11). We predicated the potential targets of miR-17-3P using miRDB and TargetScan databases, and found that there is possible binding site of miR-17-3P on 3'-UTR of Par4 (Fig. 3A). To identify the prediction and elucidate the underlying mechanism by which miR-17-3P regulates CRC cells survival, we conducted the luciferase reporter assay in SW480 and LoVo cells. As seen in Fig. 3A, the wild or mutant 3'-UTR of Par4 were cloned into luciferase vector, respectively. Luciferase activities of wild Par4-3'UTR were strongly reduced by miR-17-3P mimic, but there was no difference in the luciferase activities of mutant Par4-3'UTR in CRC cells (Fig. 3B).
Furthermore, we determined that the expression of Par4 in CRC cells was up-regulated by miR-17-3P suppression and reduced by miR-17-3P mimic (Fig. 3C). In summary, these data imply that Par4 is direct target of miR-17-3P in CRC cells.

Par4 is involved in the miR-17-3P-mediated regulation of CRC cells survival. Previous studies reported that Par4 is a pro-apoptotic factor and is required for cell apoptosis (16). To identify whether Par4 is involved in the miR-17-3P-mediated regulation of CRC cells survival, we further employed a Par4 plasmid to specifically induce the expression of Par4 in both SW480 and LoVo cells. As seen in Fig. 4A, efficiency of Par4 plasmid was confirmed by RT-PCR assay. Par4 expression was strongly enhanced by the specific recombinant plasmid in both CRC cells.

And Par4 expression reduced the proliferation of CRC cells induced by miR-17-3P, as shown in Fig. 4B. On the contrary, miR-17-3P inhibited apoptosis of SW480 and LoVo cells was reversed by Par4 compared to the control (Fig. 4C). These findings indicate that miR-17-3P regulated CRC cells survival by targeting Par4.

\section{Discussion}

Endogenous miRNAs have crucial roles as key regulators of gene expression in the physiological or pathological processes of human development and diseases (17). Differential expression of miRNAs have been reported in previous studies about $\mathrm{CRC}$, and the dysregulation of miRNAs has been considered to play positive or negative roles in colorectal carcinogenesis, 


\section{A miR-17-3p 3'-GAUGUUCACGGGAGUGACGUCA-5' \\ Par4-wt \\ Par4-mut
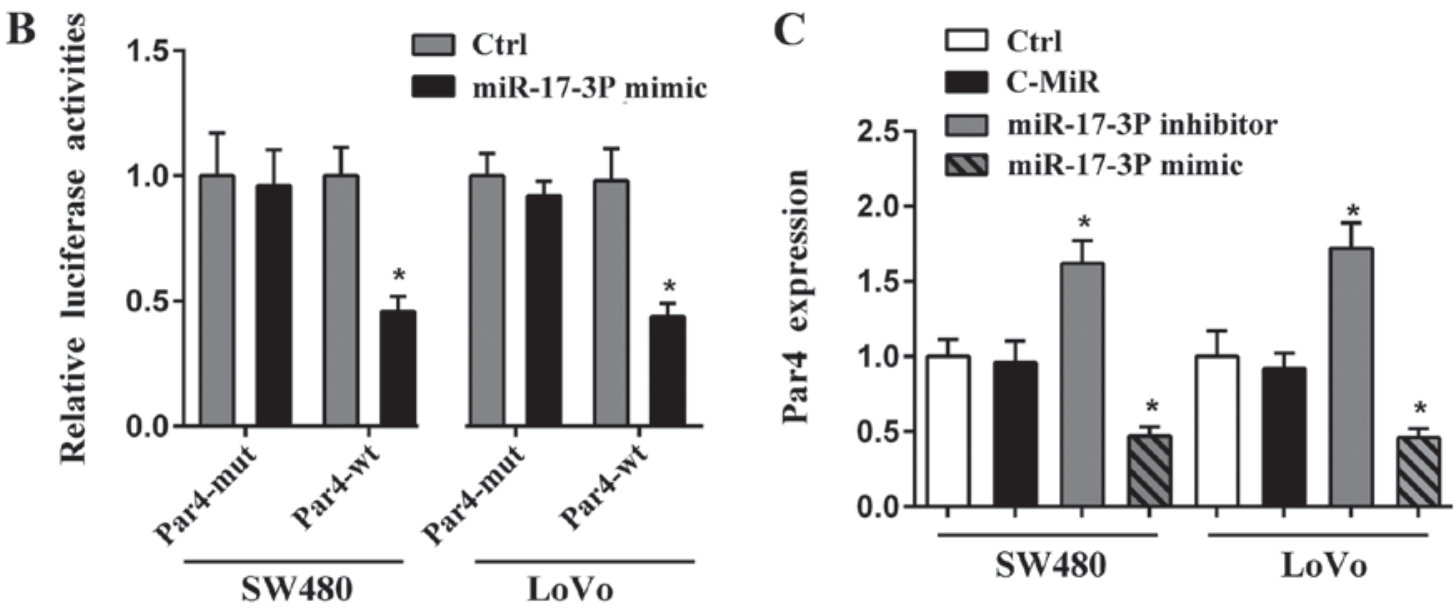

Figure 3. Par4 is the target of miR-17-3P. (A) Potential binding sites of miR-17-3P in 3'-UTR of Par4 mRNA. (B) Luciferase activities were measured in SW480 and LoVo cells after transfection by wild Par4-3'UTR (Par4-wt) or mutant Par4-3'UTR (Par4-mut). (C) The expression of Par4 in SW480 and LoVo cellss was examined by RT-PCR assay ( $\mathrm{P}<0.05$ vs. Ctrl).
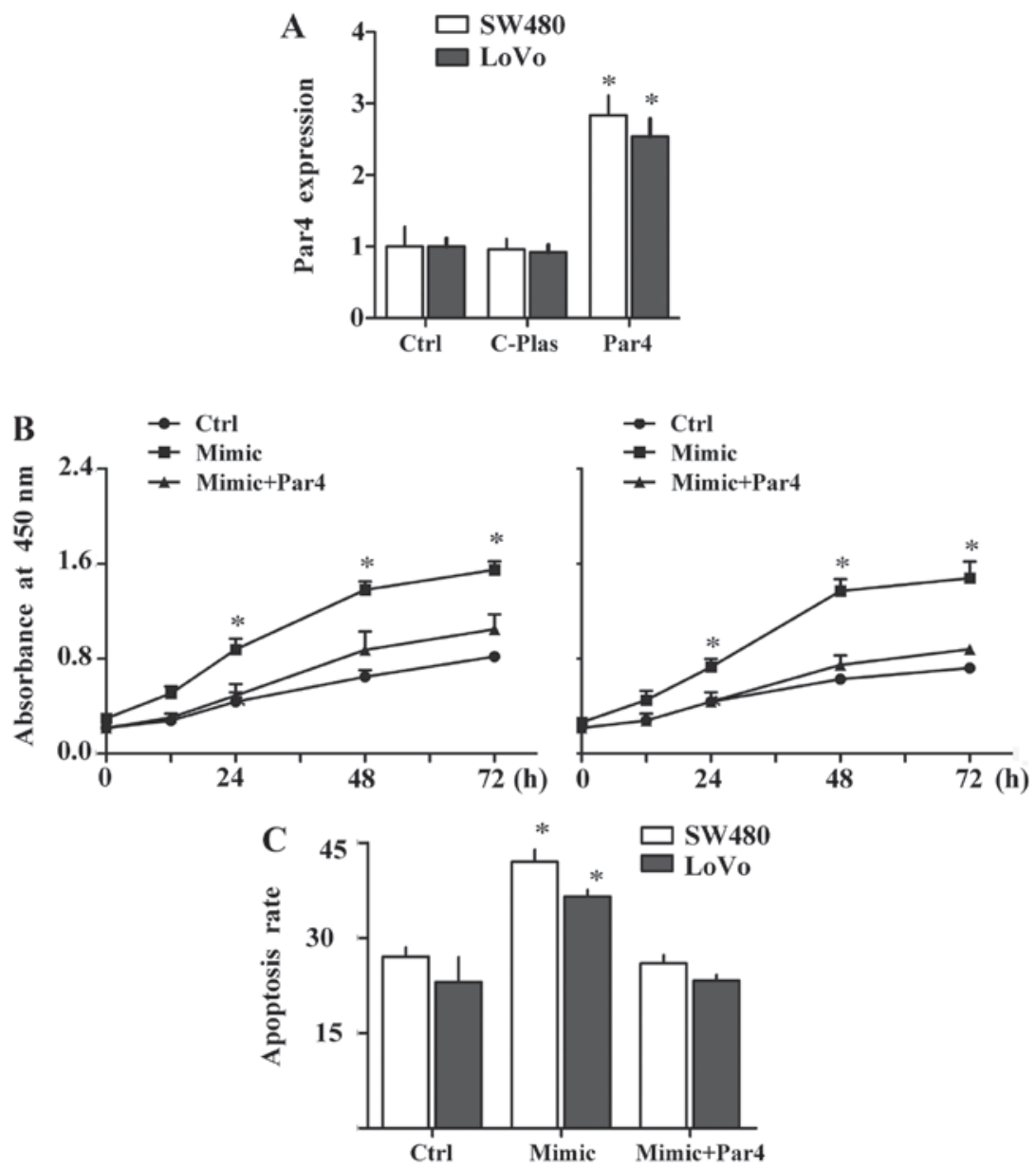

Figure 4. Par4 is involved in the miR-17-3P-mediated regulation of colorectal cancer cells survival. SW480 and LoVo cells were transfected with Par4 plasmid or negative plasmid (C-Plas). (A) Expression of Par4 was tested by RT-PCR assay in both CRC cells. Cells proliferation (B) and apoptosis (C) were then measured in SW480 and LoVo cells transfected with Par4 plasmid in absence or presence of miR-17-3P mimic ("P<0.05 vs. Ctrl). 
including miR-29a, miR-135b, miR-18b, miR-124, miR-222 and miR-31 (18-20).

miR-17-3P is derived from the miR-17 miRNA cluster, and has different roles in the physiological processes of many human diseases, such as development and remodeling of heart and lung, spinal neural progenitor patterning and cardiac fibroblast senescence $(16,21)$. Dysfunction of miR-17-3P also exerts a tumor oncogenic role in tumorigenesis. Shan et al (22) reported that the passenger strand miR-17-3p was largely expressed in transgenic mice and could induce the development of hepatocellular carcinoma by targeting PTEN and GalNT7 via various signal pathways. In another study, the increase in miR-17-3p expression was found to be associated with the stress response in glioblastoma cells. miR-17-3p could inhibit cell proliferation and drug-resistance in glioblastoma cells by repressing MDM2 levels (23). In human CRC, miR-17-3p expression was also identified to be associated with the tumorigenesis of CRC, and is involved in diagnosis as well as prognosis of CRC $(24,25)$. Herein, we demonstrated a novel role of miR-17-3p in CRC. miR-17-3p expression is significantly up-regulated in tumor tissues of patients with CRC and cultured cells. The dysregulation of miR-17-3p is associated with the regulation of survival of CRC cells by promoting cell proliferation and inhibiting apoptosis, contributing to the human CRC tumorigenesis.

Par4 is encoded by the Pawr gene and also known as PAWR. In various tissues, Par4 could interact with WT-1 protein and has an important role in cell apoptosis (26). As a tumor suppressor, Par4 is has been identified to be expressed at low levels in multiply cancers, and is correlated with the tumorigenesis of cancers (27). By online bioinformatic prediction and luciferase reporter assay, we confirmed that there is a binding site of miR-17-3P in Par4 3'-UTR, and Par4 is a direct target of miR-17-3p in human CRC cells. The expression level of Par4 was significantly repressed by miR-17-3p in human CRC cells, suggesting the involvement of miR-17-3p and Par4 in human CRC tumorigenesis. Our result is consistent with a previous report, in which miR-17-3p inhibits cardiac aging and cardiac fibroblast cellular senescence as a negative modulator by targeting Par4 and regulating the downstream proteins, including FAK, CEBPB, vimentin, N-cadherin, Oct4 and Sca-1 (16).

In previous studies, Par4 has been reported to be a crucial regulator of tumor cell survival. Induced Par4 expression could enhance the death in some cancer cells, and the down-regulation of Par4 expression might be a prognostic factor in cancer (28). Herein, we also found the involvement of miR-17-3p and Par4 in human CRC cells survival. Par4 expression reduced the proliferation of CRC cells induced by miR-17-3P. On the contrary, miR-17-3P inhibited apoptosis of SW480 and LoVo cells was reversed by Par4 compared to the control. These findings indicate that miR-17-3P regulated CRC cells survival by targeting Par4.

In conclusion, we determined that increased miR-17-3P level plays crucial role in CRC cells survival by targeting Par4, contributing to colorectal carcinogenesis. This may indicate a novel finding about human CRC progression.

\section{References}

1. Haigis KM: Molecular pathogenesis of colorectal cancer. Anticancer Res 11: 609-633, 2014.
2. Fearon ER: Molecular genetics of colorectal cancer. Ann Rev Pathol Mechanisms Dis 6: 479-507, 2011.

3. Ng EK, Chong WW, Jin H, Lam EK, Shin VY, Yu J, Poon TC, Ng SS and Sung JJ: Differential expression of microRNAs in plasma of patients with colorectal cancer: A potential marker for colorectal cancer screening. Gut 58: 1375-1381, 2009.

4. Zhang Z, Tang H, Wang Z, Zhang B, Liu W, Lu H, Xiao L, Liu X, Wang R, Li X, et al: MiR-185 targets the DNA methyltransferases 1 and regulates global DNA methylation in human glioma. Mol Cancer 10: 124, 2011.

5. Farazi TA, Hoell JI, Morozov P and Tuschl T: MicroRNAs in human cancer. In: MicroRNA Cancer Regulation Springer, ppl-20, 2013.

6. Calin GA and Croce CM: MicroRNA signatures in human cancers. Nat Rev Cancer 6: 857-866, 2006

7. Luo X, Burwinkel B, Tao S and Brenner H: MicroRNA signatures: Novel biomarker for colorectal cancer? Cancer Epidemiol Biomarkers Prev 20: 1272-1286, 2011.

8. Koga Y, Yasunaga M, Takahashi A, Kuroda J, Moriya Y, Akasu T, Fujita S, Yamamoto S, Baba H and Matsumura Y: MicroRNA expression profiling of exfoliated colonocytes isolated from feces for colorectal cancer screening. Cancer Prev Res (Phila) 3: $1435-1442,2010$

9. Bandres E, Agirre X, Bitarte N, Ramirez N, Zarate R, Roman-Gomez J, Prosper F and Garcia-Foncillas J: Epigenetic regulation of microRNA expression in colorectal cancer. Int $\mathrm{J}$ Cancer 125: 2737-2743, 2009.

10. Olive V, Jiang I and He L: mir-17-92, a cluster of miRNAs in the midst of the cancer network. Int J Biochem Cell Biol 42: 1348-1354, 2010.

11. Hebbar N, Shrestha-Bhattarai $\mathrm{T}$ and Rangnekar VM: Cancer-selective apoptosis by tumor suppressor par-4. Adv Exp Med Biol 818: 155-166, 2014.

12. Pereira MC, de Bessa-Garcia SA, Burikhanov R, Pavanelli AC, Antunes L, Rangnekar VM and Nagai MA: Prostate apoptosis response- 4 is involved in the apoptosis response to docetaxel in MCF-7 breast cancer cells. Int J Oncol 43: 531-538, 2013.

13. Da Bessa-Garcia SA, Pereira M and Nagai MA: Regulation of PAWR expression by estrogen and growth factors in breast cancer cells. Int J Mol Med: 24, 2009.

14. Yang K, Shen J, Chen SW, Qin J, Zheng XY and Xie LP: Upregulation of PAWR by small activating RNAs induces cell apoptosis in human prostate cancer cells. Oncol Rep 35: 2487-2493, 2016.

15. Zhang Y, Zheng D, Xiong Y, Xue C, Chen G, Yan B and Ye Q: miR-202 suppresses cell proliferation in human hepatocellular carcinoma by downregulating LRP6 post-transcriptionally. FEBS Lett 588: 1913-1920, 2014.

16. Du WW, Li X, Li T, Li H, Khorshidi A, Liu F and Yang BB: The microRNA miR-17-3p inhibits mouse cardiac fibroblast senescence by targeting Par4. J Cell Sci 128: 293-304, 2015.

17. Bartel DP: MicroRNAs: Genomics, biogenesis, mechanism, and function. Cell 116: 281-297, 2004.

18. Xi Y, Formentini A, Chien M, Weir DB, Russo JJ, Ju J, Kornmann M and Ju J: Prognostic values of microRNAs in colorectal cancer. Biomarker Insights 2: 113-121, 2006.

19. Motoyama K, Inoue H, Takatsuno Y, Tanaka F, Mimori K, Uetake H, Sugihara K and Mori M: Over- and under-expressed microRNAs in human colorectal cancer. Int J Oncol 34: 1069-1075, 2009.

20. Lanza G, Ferracin M, Gafà R, Veronese A, Spizzo R, Pichiorri F, Liu CG, Calin GA, Croce CM and Negrini M: mRNA/microRNA gene expression profile in microsatellite unstable colorectal cancer. Mol Cancer 6: 54, 2007.

21. Chen JA, Huang YP, Mazzoni E, Tan GC, Zavadil J and Wichterle H: Mir-17-3p controls spinal neural progenitor patterning by regulating Olig2/Irx3 cross-repressive loop. Neuron 69: 721-735, 2011.

22. Shan SW, Fang L, Shatseva T, Rutnam ZJ, Yang X, Du W, Lu WY, Xuan JW, Deng Z and Yang BB: Mature miR-17-5p and passenger miR-17-3p induce hepatocellular carcinoma by targeting PTEN, GalNT7 and vimentin in different signal pathways. J Cell Sci 126: 1517-1530, 2013.

23. Li H and Yang BB: Stress response of glioblastoma cells mediated by miR-17-5p targeting PTEN and the passenger strand miR-17-3p targeting MDM2. Oncotarget 3: 1653-1668, 2012.

24. Faltejskova P, Bocanek O, Sachlova M, Svoboda M, Kiss I, Vyzula R and Slaby O: Circulating miR-17-3p, miR-29a, miR-92a and miR-135b in serum: Evidence against their usage as biomarkers in colorectal cancer. Cancer Biomark 12: 199-204, 2012. 
25. Xie Y and Emergency DO: Application of miRNA-17-3p combined with CEA in the diagnosis and treatment of colorectal cancer. J Clin Med Practice, 2014.

26. Chen X, Sahasrabuddhe AA, Szankasi P, Chung F, Basrur V, Rangnekar VM, Pagano M, Lim MS and Elenitoba-Johnson KS: Fbxo45-mediated degradation of the tumor-suppressor Par-4 regulates cancer cell survival. Cell Death Differ 21: 1535-1545, 2014.
27. Goswami A, Burikhanov R, de Thonel A, Fujita N, Goswami M, Zhao Y, Eriksson JE, Tsuruo T and Rangnekar VM: Binding and phosphorylation of par-4 by Akt is essential for cancer cell survival. Mol Cell 20: 33-44, 2005.

28. Ranganathan P and Rangnekar VM: Regulation of cancer cell survival by par-4. Ann N Y Acad Sci 1059: 76-85, 2005. 